\title{
"Plus peur de l'hiver que du diable » : des immigrants aux hivernants canadiens-français à Palm Beach (Floride),
} 1945-1997

\section{Serge Dupuis}

Volume 63, numéro 4, printemps 2010

URI : https://id.erudit.org/iderudit/1006007ar

DOI : https://doi.org/10.7202/1006007ar

Aller au sommaire du numéro

Éditeur(s)

Institut d'histoire de l'Amérique française

ISSN

0035-2357 (imprimé)

1492-1383 (numérique)

Découvrir la revue

Citer cet article

Dupuis, S. (2010). « Plus peur de l'hiver que du diable » : des immigrants aux

hivernants canadiens-français à Palm Beach (Floride), 1945-1997. Revue

d'histoire de l'Amérique française, 63(4), 465-495.

https://doi.org/10.7202/1006007ar

\section{Résumé de l'article}

Cet article explore la constitution d'une communauté immigrante canadienne-française à Palm Beach en période d'après-guerre. La venue d'un contingent grandissant de touristes et d'hivernants durant les années 1960 et 1970 transforma cette communauté qui avait misé autrefois sur l'intégration aux États-Unis et visait désormais la création d'un contexte francophone pendant l'hiver, bien en lien avec la mère patrie. Si les préoccupations économiques se trouvent au centre de cette migration, la « peur » de l'hiver se hisse également au rang des constantes en Floride canadienne-française. 


\section{“Plus peur de l'hiver que du
diable» $:$ des immigrants aux
hivernants canadiens-français à
Palm Beach (Floride), I945-1997}

Serge Dupuis

Département d'Histoire

University of Waterloo

RÉSUMÉ - Cet article explore la constitution d'une communauté immigrante canadienne française à Palm Beach en période d'après-guerre. La venue d'un contingent croissant de touristes et d'hivernants durant les années 1960 et 1970 changea profondément cette communauté qui avait misé autrefois sur l'intégration aux États-Unis et visait désormais la création d'un contexte francophone pendant l'hiver, bien en lien avec la mère patrie. Si les préoccupations économiques se trouvent au centre de cette migration, la «peur» de l'hiver se hisse également au rang des constantes en Floride canadienne-française.

ABSTRACT - This article explores the foundation of a French Canadian immigrant community in Palm Beach, Florida in the Post-war years. The arrival of an ever-growing contingent of tourists and Snowbirds during the 1960s and 1970s profoundly changed this community. Formerly focused on integration into American society, it now set out to create a francophone context well connected to French Canada. If economic imperatives often motivated this migration, the «fear» of winter also appears to be an element of continuity in French Canadian Florida.

1. Tiré du mémoire de maîtrise de l'auteur, L'émergence d'une Floride canadienne-française: l'exemple de la communauté de Palm Beach, 1910-2010, mémoire de maîtrise (histoire), Université d'Ottawa, 2009. 


\section{INTRODUCTION ${ }^{2}$}

Durant son apostolat, $\mathrm{M}^{\mathrm{gr}}$ Ignace Bourget dépêcha trois missionnaires montréalais en Floride afin d'évangéliser les Séminoles et de prêter mainforte à l'Église locale ${ }^{3}$. En janvier 1868, il s'empressa de féliciter le missionnaire Augustin Laverdière de «son amour pour la sainte Église, en se consumant de travaux pour la répandre et l'exalter dans les lieux les plus lointains ${ }^{4} »$. Personne à l'époque n'aurait cru que ce «lieu lointain» deviendrait une destination privilégiée par les vacanciers, immigrants et hivernants canadiens-français le siècle suivant. L'abbé Henri-Raymond Casgrain et le premier ministre canadien Wilfrid Laurier furent parmi les premiers touristes illustres à y séjourner respectivement en 1880 et en $1902^{5}$. Certains travailleurs y œuvrèrent dans l'industrie forestière du golfe du Mexique ou dans les vergers des terres intérieures.

Ces migrations temporaires ont suscité quelques tentatives de colonisation canadienne-française, dont la colonie acadienne du lac Okeechobee $(1924)^{6}$ et la colonie franco-américaine de Bélandville (1930-1936) Toutefois, ces colonies ne se comparent en rien aux « Petits Canadas» qui ont rassemblé près de 900000 Canadiens français en Nouvelle-Angleterre entre 1840 et $1930^{8}$. En 1940, le recensement américain comptait 877 résidants canadiens-français ${ }^{9}$. Même si leur nombre s'élevait à quelques

2. L'auteur remercie d'abord Michel Bock, son directeur de thèse, ainsi que Godefroy Desrosiers-Lauzon, Anne Gilbert, Yves Frenette, les lecteurs anonymes et le comité de rédaction de la RHAF pour leurs critiques d'une version antérieure de cet article; l'appui financier du Conseil de recherches en sciences humaines du Canada, de l'Université d'Ottawa (la Faculté des études supérieures, le Département d'Histoire, le Fonds Gaston-Héon, le Centre de recherche en civilisation canadienne-française) et du Conseil de la vie en Amérique française; les Canadiens français de Palm Beach; et sa famille pour leur amour et intérêt.

3. François Beaudin, "Prêtres de Montréal en mission aux États-Unis (1836-1876)», Revue d'histoire de l'Amérique française, 21, 4 (1968): 792.

4. Ignace Bourget, Lettre à Augustin Laverdière, 1868, ibid., 802.

5. Yvan Lamonde, Ni sans eux, ni avec eux: Le Québec et les États-Unis (Montréal, Nuit blanche Éditeur, 1996), 45; Godefroy Desrosiers-Lauzon, «À l'envers de l'hiver: le voyage en Floride et les identités canadienne et québécoise », Histoire sociale, 39, 77 (automne 2006): 111-112.

6. "Conners Sells 1,000 Acres to Florida Colony of French Canadians», The Palm Beach Post, 22 janvier 1924, Fonds Populations (Canadians in Florida), Historical Society of Palm Beach County Archives (HSPBCA), West Palm Beach (Floride).

7. Laura Lee Scott, Belandville: A French Canadian Colony in West Florida (Bagdad, Floride) (Pantagonia Press, 2005).

8. Voir, entre autres: Yves Roby, Les Franco-Américains de la Nouvelle-Angleterre, rêves et réalités (Sillery, Septentrion, 2000).

9. «Florida + Canadian + French Canadian + 1940», University of Virginia Library Historical Census Browser, http://fisher.lib.virginia.edu/collections/stats/histcensus [site consulté le 21 juillet 2010]. 
milliers (le U.S. Census prenait seulement en compte les résidants permanents et immigrants francophones nés au Canada), les Canadiens français en Floride demeuraient peu nombreux.

Cette marginalité explique sans doute le mutisme de l'historiographie sur cette présence. Les géographes Louis Dupont et Rémy Tremblay ont consacré des recherches aux Québécois en Floride, mais l'aperçu de la migration et de l'installation de ces derniers fut sommaire ${ }^{10}$. Un premier historien, Godefroy Desrosiers-Lauzon, consacra une thèse au phénomène des hivernants nord-américains en Floride, mais il ne s'est pas intéressé aux changements dans la composition des communautés canadiennes-françaises autrefois à prédominance immigrante et touristique et recomposées d'hivernants et de touristes plus récemment. Si nous nous sommes intéressé à la Floride, c'est en raison de la croissance spectaculaire du nombre de Canadiens français qu'on y observe au lendemain de la Deuxième Guerre mondiale. La population résidente passa de 1808 en 1950 à environ 50000 en $1970^{11}$. De plus, le nombre de touristes canadiens passa de $245000^{12}$ en 1960 à un million en 1970 dont 30 à $40 \%$ étaient francophones. En peu de temps, des communautés immigrantes et vacancières ont surgi sur la côte Atlantique. Si bien que le journaliste Pierre Paquette remarquait, lors d'une visite à «Miami P.Q. ${ }^{13}$ » en février 1967, que les touristes et résidants pouvaient profiter d'une gamme complète d'activités et de services en français.

Cet article a pour objet la communauté canadienne-française de Palm Beach du début de l'importante migration vers la Floride après la

10. Louis Dupont et Marie Dussault, «La présence francophone en Floride : un portrait», Vie française, 36, 10-11-12 (octobre-décembre 1982): 5-22; Louis Dupont, "Le déplacement et l’implantation des Québécois en Floride», Vie française, 36, 10-12 (octobre-décembre 1982): 23-33; Rémy Tremblay, Floribec : espace et communauté (Ottawa, Presses de l'Université d'Ottawa, 2006).

11. " $1950+1960+1970+1980+1990+$ Social and Economic Characteristics + Florida", Census of Population and Housing - Decennial Censuses, www.census.gov/prod/www/abs/ decennial/index.htm [site consulté le 21 juillet 2010]. L'État comptait 114615 résidants d'ascendence canadienne en 1970. L’importance de la présence canadienne-française en cette année n’est pas précisée. En 1950, les 1808 Canadiens français représentaient $12 \%$ de la population canadienne s'élevant à 14992 personnes. En 1990, les 110221 résidants canadiens-français représentaient 71\% de la population canadienne de 155073 âmes. Puisque la proportion canadienne-française augmente d'un recensement à l'autre, nous estimons qu'elle représenterait environ $32 \%$ de la population canadienne totale. C'est ainsi que nous arrivons à l'estimation d'une population canadiennefrançaise de 50000 en 1970.

12. G. Desrosiers-Lauzon, "À l'envers de l'hiver... », loc. cit., 112.

13. Pierre Paquette, «Les Québécois en Floride», Aujourd’hui, 4 avril 1967, Archives de RadioCanada, http: / / archives.radio-canada.ca/art_de_vivre/voyage/clips / 16233/ [site consulté le 21 juillet 2010]. 
Deuxième Guerre mondiale jusqu’à son apogée à la fin du siècle. Sans être soumis à un cadre théorique particulier, notre analyse vise à mettre en lumière l'historiographie pertinente sur le tourisme, les migrations, la Franco-Américanie ainsi que le Canada français tout en employant les théories de l'historien Paul-André Rosental ${ }^{14}$ sur les «migrations de maintien» et les "migrations de rupture» et celles du sociologue Joseph Yvon Thériault sur le spectre entre les intentions ethnique, nationalitaire ${ }^{15}$ et nationale ${ }^{16}$.

Dans leurs cas respectifs (les migrations intrafrançaises et les communautés francophones en milieu minoritaire du Canada), ces chercheurs distinguent les communautés minoritaires qui visent l'intégration à leur société d'accueil de celles qui choisissent de maintenir des liens avec la société majoritaire de laquelle elles sont issues. Cette ambition de "faire société» exige toutefois un développement institutionnel ainsi qu'un réseautage complexe pour qu'elle soit maintenue. Dans le cas des migrations récentes, il s'agit de réaliser une «migration de maintien». Entre les deux pôles, il existe un spectre au milieu duquel se trouve une ambition "nationalitaire», selon Thériault, qui mêle des éléments de la communauté d'acculturation et de la communauté nationale. Il va sans dire que les parcours individuels des Canadiens français en Floride se trouvent partout sur ce spectre. Cependant, l'observation du cas de Palm Beach dans la période étudiée démontre le passage d'une communauté à dominance immigrante à une communauté à dominance hivernante, mais aussi d'une communauté principalement ethnique à une communauté investie d'un caractère nationalitaire. Plus tard, ce terme sera nuancé puisque mobilisé à l'extérieur du Canada. Notons surtout qu'une mutation s'effectua à la fin des années 1970 qui transforma le visage de la Floride canadienne-française. La solidarité retrouvée entre Canadiens français du Québec, de l'Ontario et de la Nouvelle-Angleterre sera explorée comme un autre caractère bien particulier de cet îlot francophone en Amérique.

Dans le but de rendre justice à l'exemple de Palm Beach, la deuxième région francophone en importance de la Floride après Hollywood, nous employons une gamme variée de sources écrites et orales. Les archives

14. Paul-André Rosental, «Maintien/rupture : un nouveau couple pour l'analyse des migrations", Annales ESC, 6 (novembre-décembre 1990): 1403-1431.

15. Joseph Yvon Thériault, «Entre la nation et l'ethnie: Sociologie, société et communautés minoritaires francophones", Sociologie et sociétés, 26, 1 (printemps 1994): 15-32.

16. Joseph Yvon Thériault, Faire société. Société civile et espaces francophones (Sudbury, Prise de Parole, 2007). 
du Club canadien-français d'Amérique (CCFA) et celles de la Communauté catholique d'expression française de Lake Worth (CCEFLW) rappellent les parcours particuliers de ces associations. Les archives de la Historical Society of Palm Beach County (HSPBC) furent aussi utiles pour découvrir l'ancienneté de la présence canadienne-française dans la région. Des études secondaires tout comme les renseignements de Statistique Canada et des recensements décennaux américains ont permis l'évaluation de l'importance des migrations et de l'établissement en Floride. Les études d'Yves Roby, d'Yves Frenette et de Jean Lamarre sur les Canadiens français aux États-Unis ont offert des repères théoriques. Enfin, les bases de données Biblio branchée et de Canadian Newstand ont signalé plusieurs articles de presse sur les relations canado-floridiennes depuis les années 1970.

À l'inverse des communautés de la Nouvelle-Angleterre, aucune élite intellectuelle ne s'est installée en Floride pour accompagner l'établissement des Canadiens français, ce qui se solde par la rareté des traces laissées sur les efforts et les défis de cette population. Faute d'un journal, d'une paroisse ou d'une mutuelle présents pendant la période étudiée, nous avons recensé les récits de vie de 25 migrants (seize nés au Québec, cinq en Ontario et quatre aux États-Unis) par l'entremise d'entrevues effectuées en janvier 2008 et en janvier 2009. Il semblait de mise de capter des témoignages "qui [allaient] disparaître à tout jamais ${ }^{17}$ » en raison de l'âge avancé de plusieurs migrants. En empruntant des stratégies d'entrevues semidirigées du collectif The Oral History Reader ${ }^{18}$, des thèmes communs sur la migration, l'établissement et les visions du passé furent abordés tout en privilégiant aussi les nuances des parcours respectifs. En dernier lieu, un sondage fut aussi distribué auprès de 35 hivernants en 2008 qui générèrent 22 réponses. Son apport fut de nuancer les parcours des immigrants.

Ce texte abordera la Floride canadienne-française et le cas de Palm Beach en trois parties: la reprise de l'immigration vers les États-Unis entre 1945 et 1968, la formation d'une communauté ethnique à Palm Beach entre 1969 et 1977 et sa transformation grâce à une population toujours grandissante d'hivernants entre 1977 et 1997. La chute récente de l'attrait floridien ne sera pas abordée puisqu'elle mériterait un article à elle seule.

17. Jacques Rouillard, Ah les États! Les travailleurs canadiens-français dans l'industrie textile de la Nouvelle-Angleterre d'après le témoignage des derniers migrants (Montréal, Boréal Express, 1985), 13.

18. Robert Perks et Alistair Thomson, The Oral History Reader (Londres/ New York, Routledge, 2006). 


\section{LE SUD-EST DE LA FLORIDE COMME NOUVEAU PÔLE DE L’AMÉRIQUE FRANÇAISE, 1945 - 1968}

Entre 1812 et 1914, les guerres du vieux continent, la disponibilité de nouvelles terres et l'industrialisation incitèrent 55 millions de personnes à émigrer aux États-Unis. Parmi eux, environ 900000 Canadiens français se dirigèrent principalement vers les villes manufacturières de la NouvelleAngleterre. La Crise économique des années 1930 et la relance de la colonisation agricole du Nord québécois et ontarien mirent fin à l'ampleur de cette ruée vers le Sud. Cette pause fut cependant de courte durée: le Braceros Program (1944) facilita l'installation de travailleurs continentaux de la construction et agricoles et l'Immigration and Nationality Act (1952) élimina certains critères discriminants tels la nationalité pour accepter ou non les immigrants ${ }^{19}$. L'immigration reprit donc de plus belle autant parmi les Européens que les Canadiens.

Entre 1950 et 1960, le nombre de Canadiens habitant la Floride a quadruplé (passant de 15000 à 58 000) et doublé pendant la décennie suivante pour atteindre 114000 personnes en $1970^{20}$. L'augmentation du contingent canadien-français est encore plus impressionnant: d'une population de 1800 en 1950, il s'éleva à environ 50000 résidants permanents en $1970^{21}$; une multiplication par 25 en deux décennies. Leur présence augmentait le nombre de Floridiens dont la langue maternelle était le français à $138000^{22}$. Par contre, la nouvelle loi d'immigration de 1965 (le Preference System) privilégia la réunification des familles des Américains naturalisés et mit fin à l'exemption du système de quotas dont bénéficiait les Canadiens. Puisque le taux de naturalisation canadienne était parmi les plus bas au pays ${ }^{23}$, le Preference System, tout comme la Crise économique

19. Robert Barde, Susan B. Carter et Richard Sutch, dir., "Chapter Ad - International Migrations», Historical Statistics of the United States. Earliest Times to the Present. Millennial Edition. Volume One. Part A. Population (New York, Cambridge University Press, 2006), I-527.

20. «1950 + 1960 + 1970 + Social and Economic Characteristics + Florida», Census of Population and Housing - Decennial Censuses, www.census.gov/prod/www/abs/decennial/ index.htm [site consulté le 21 juillet 2010].

21. Douze pour cent de la population canadienne en Floride était canadienne-française en 1950. En 1990, le même taux était de 73 pour cent. Si le taux avait cru de façon constante pendant l'intervalle, le poids des Canadiens français parmi la population canadienne aurait été de 43 pour cent en 1970.

22. R. Tremblay, Floribec..., op. cit., 16-18; «Florida - Table 71. Ethnic Characteristics by Metropolitan and Non-Metropolitan Residence: 1970 », US Census of Population (Washington, U.S. Census Bureau, 1972), 11/249.

23. En 1990, 11 pour cent des résidents permanents canadiens étaient naturalisés. Seuls les résidents permanents britanniques connaissaient un taux plus faible de naturalisation; R. Barde et al., "Chapter Ad - International Migrations...», op. cit., I-647. 
des années 1970, ont rendu l'émigration vers les États-Unis plus complexe et moins attrayante qu'auparavant. En conséquence, le taux d'émigration canadienne aux États-Unis chuta de $73 \%$ au cours de la décennie suivante.

La Floride avait, quant à elle, entrepris une vaste campagne de relations publiques, dès la fin des années 1930, pour construire une image populaire de la Floride ${ }^{24}$ l'associant au plaisir, à la chaleur et à la jeunesse ${ }^{25}$. Depuis les années 1920, des tracés routiers reliaient l’Ontario et le Québec respectivement aux côtes ouest et est de la Floride; une réalité qui explique la concentration des Canadiens français sur la côte Atlantique et des Canadiens anglais sur les berges du golfe du Mexique. L'historien Eric Jarvis remarque que, dès les années 1930, des Canadiens français travaillaient dans les chantiers de canalisation de Miami ${ }^{26}$. Louis Dupont constate que les travailleurs et anciens combattants franco-américains s'installèrent au nord de Miami tant pour des raisons économiques que climatiques $^{27}$. De plus, la Floride restait l'unique station balnéaire subtropicale accessible par les routes. Dans le contexte de prospérité grandissante d'après-guerre et dans un État où les taux d'imposition et les prix des marchandises étaient parmi les plus modiques du continent, la Floride devenait de plus en plus invitante ${ }^{28}$. Pour se débarrasser des moustiques et remédier à l'humidité qui accablaient la région, des fabricants de pesticides et de climatiseurs rendirent leurs produits abordables au consommateur moyen ${ }^{29}$. Pour sa part, l'avènement des Interstate américaines (les autoroutes à chaussées séparées), réduisit la durée du trajet vers la Floride de moitié depuis le Canada central ${ }^{30}$.

En banlieu de Miami, Hollywood charmait surtout des Montréalais ${ }^{31}$, mais le secteur de Lake Worth à Palm Beach rassemblait davantage de

24. Gary R. Mormino, Land of Sunshine, State of Dreams: A Social History of Modern Florida, 1950-2000 (Gainesville, University Press of Florida, 2005), 179.

25. Raymond Arsenault, "Is There a Florida Dream?», Forum (été 1994): 25.

26. Eric Jarvis, «Florida’s Forgotten Ethnic Culture: Patterns of Canadian Immigration, Tourism and Investment since 1920", Florida Historical Quarterly, 81, 2 (automne 2002): 186.

27. L. Dupont, «Le déplacement et l’implantation des Québécois... », loc. cit., 23-33.

28. David Nelson, "When Modern Florida was Born: Florida at the World Fairs and on the World Stage in the 1930s", Florida Historical Society Annual Meeting, communication libre, Pensacola (Floride), 22 mai 2009.

29. Raymond Arsenault, «The End of Long Hot Summer: The Air Conditioner and Southern Culture", Journal of Southern Culture, 50, 4 (novembre 1984): 597-628.

30. G. Mormino, Land of Sunshine, State of Dreams..., op. cit., 78.

31. L. Dupont, «Le déplacement et l’implantation des Québécois... », loc. cit., 24. 
migrants venus de l'Estrie et de la Beauce ${ }^{32}$. Pour la plupart âgés d'une vingtaine d'années, les immigrants faisaient référence au climat, aux meilleures conditions de travail ou au meilleur pouvoir d'achat pour justifier leur déplacement. Par exemple, Conrad Roy de Rock Island (Québec) constata que le travail de la mécanique n'était pas profitable au Québec pendant trois saisons sur quatre, mais il était surtout ravi du fait que les mécaniciens de la Floride travaillaient «en chemise blanche!» Sans gelée, gadoue ou sel, les mécaniciens seraient "fous de travailler [ailleurs] ${ }^{33}$ " qu'en Floride. À la suite de son premier séjour à l'hiver de 1958, il s'installa plus tard la même année à Lake Worth où il exploita son propre garage pendant 45 ans, jusqu'à sa retraite. Quant à elle, Rose Brousseau de Montréal rejoignit ses parents qui hivernaient en Floride depuis quelques années en 1959. Elle fut charmée au point de téléphoner à son mari au Québec pour lui annoncer qu'ils s'établiraient à Miami dans les plus brefs délais. De retour au Canada, elle soumit une demande de permis de travail et le reçut cinq semaines plus tard ${ }^{34}$.

Joseph Asselin de Stanstead (Québec) prit congé pendant l'hiver 1964 pour travailler à Surfside. Trois mois plus tard, il rentra dans un Québec enneigé, démissionna de son poste au Zeller’s de Granby et émigra en Floride l'automne suivant. Il possédait un niveau d'anglais approximatif, "pas pour tenir une bonne conversation", mais assez tout de même pour connaitre «les termes du magasin [qui] étaient tous bilingues [à Granby] ${ }^{35}$ ». Sa conjointe Nicole Bureau fut charmée, elle aussi, par son premier séjour et fit des démarches pour que sa formation d'infirmière soit reconnue en Floride. "On n'avait pas besoin de refaire de tests pour la licence [sic], constatait-elle. C'était juste un paquet de paperasses à remplir ${ }^{36}$ ".

Originaires de la Beauce, Roland et Simone Doyon s'intéressaient peu à l'agriculture que leurs ancêtres avaient pratiquée depuis des générations. Ils partirent donc pour le Maryland en 1964 où M. Doyon décrocha un emploi dans le secteur de la construction. Le père de Roland aurait voulu que son fils reprenne la ferme, «mais quand j'suis monté icitte, c'était final ${ }^{17}$ », expliquait-il. Ils déménagèrent à Palm Beach lorsque de meilleures occasions professionnelles se présentèrent en 1968. Non seulement le

32. Sondage auprès des Canadiens français de Palm Beach, texte inédit, janvier 2008.

33. Conrad Roy, Entrevue, Greenacres (Floride), 8 janvier 2008.

34. Rose Brousseau, Entrevue, Lake Worth (Floride), 12 janvier 2008.

35. Joseph Asselin, Entrevue, Lantana (Floride), 9 janvier 2008.

36. Nicole Bureau, Entrevue, Lantana (Floride), 9 janvier 2008.

37. Roland Doyon, Entrevue, Palm Springs (Floride), 8 janvier 2009. 
travail paraissait-il plus stable en construction aux États-Unis, mais il était encore possible d'œuvrer dans le métier dans certains États sans la formation professionnelle devenue obligatoire au Canada.

Ça prenait des cartes [professionnelles] au Canada si tu voulais rien que travailler avec un pic puis une pelle. [...] Quand j'suis arrivé aux États icitte, j'suis monté [de statut économique] dans le temps de le dire [...] Le monde qui s'en venait icitte, y se pognait une job t'suite. Y'avait de l'ouvrage partout!

Pour Simone Doyon, l'émigration en Floride représentait une façon "de faire quelque chose de bien de [sa] $\mathrm{vie}^{38}$ » avec peu d'instruction. Ajoutons finalement que les Canadiens profitaient généralement d'un préjugé favorable à leur éthique de travail.

Selon Rosental, la migration amène souvent les migrants à distinguer leur espace d'origine de leur espace d'accueil, par la caricature du pays d'origine. Les migrants l'associeront à la tradition, aux contraintes et aux particularismes, tandis que le milieu d'accueil sera synonyme de modernité, de liberté et de cosmopolitisme ${ }^{39}$. Roby remarque d'ailleurs que plusieurs immigrants canadiens-français en Nouvelle-Angleterre percevaient plus de pauvreté et de défis au Québec que dans leur pays d'adoption, même si les conditions de vie y étaient assez similaires ${ }^{40}$. Les thèses de la "grande noirceur» du Canada français avant 1960 ont largement été démenties par les historiens et l'élargissement de la prospérité y gagnait du terrain comme ailleurs en Amérique. Toutefois, les revenus légèrement inférieurs à la norme canadienne perduraient et le chômage saisonnier restait plus fréquent en milieu rural au Québec ${ }^{41}$. Qu'ils aient stigmatisé leur milieu d'origine ou idéalisé la Floride, plusieurs migrants choisirent de s'y installer puisque l'esprit d'entreprise était plus grand et la probabilité de réussite financière était plus forte.

D’autres migrants, comme Maurice Ouellette de Sudbury (Ontario), choisirent la migration temporaire dès un jeune âge. Ouellette posait du stuc et suivit une formation à Miami en 1962. Émerveillé par la beauté de la flore de la région, il décida d'y travailler l'hiver pendant l'essentiel de sa vie adulte. Dans son cas, comme dans tant d'autres, les autoroutes et

38. Simone Doyon, Entrevue, Palm Springs (Floride), 8 janvier 2009.

39. P.-A. Rosental, «Maintien/rupture...», loc. cit., 1405.

40. Y. Roby, Les Franco-Américains de la Nouvelle-Angleterre, rêves et réalités, op. cit., 27, 266-271.

41. Paul-André Linteau et al., Histoire du Québec contemporain de 1930 à nos jours (Montréal, Boréal Compact, 1989), 167-177, 204-210, 225-234, 311, 319-327, 623-627, 630; voir aussi : JeanLouis Gagnon et A. Davidson Dunton, dir., Commission royale d'enquête sur le bilinguisme et le biculturalisme. Volume 3 : Le monde du travail (Ottawa, Imprimeur de la Reine, 1969), 499-646. 
les vols d'avion plus fréquents permettaient de posséder deux domiciles à des milliers de kilomètres l'un de l'autre ${ }^{42}$.

D’autres "rêveurs" floridiens ont effectué des migrations en étapes comme le décrit Yves Frenette. Avant de pénétrer dans les États plus au sud, certains se déplaçaient vers les États limitrophes du Québec ${ }^{43}$. C'est le cas d'Albert et de Ginette, nés en Estrie, et qui émigrèrent à Richford (Vermont), respectivement en 1949 et en 1958, où les salaires étaient supérieurs à ceux du Québec. Ils déménagèrent en Floride au moment de leur retraite ${ }^{44}$. Raymond Lemay de Chartierville (Québec) émigra à Canaan (Vermont) en 1955 avant de s'installer en Floride en 1986. Pour lui, les salaires supérieurs aux États-Unis tout comme un dégoût pour le froid qui le "maganait ben gros ${ }^{45}$ » dans le travail forestier l'ont encouragé à vivre au Vermont puis en Floride. D’autres Franco-Américaines comme Denise D’Anjou du «Petit Canada » de Manchester partirent vivre à Palm Beach au milieu des années $1980^{46}$.

Avant 1960, les migrants s'intégrèrent rapidement au marché de travail et à la société américaine. Pour bon nombre d'entre eux, il s'agissait d'une migration de rupture puisqu'ils ne retournèrent jamais habiter au Canada. La vague d'immigration de Canadiens français vers la Floride ne prit cependant jamais l'ampleur de celle vers la Nouvelle-Angleterre. Malgré la sévérité de certains propos, ni la surpopulation ni la disette n'avaient incités ces migrants à trouver refuge en Floride, mais bien le désir de tenter sa chance au soleil.

\section{LA CONSOLIDATION D'UNE COMMUNAUTÉ IMMIGRANTE À PALM BEACH, 1969-1976}

Pour Bureau et Asselin, lorsque les Floridiens percevaient un accent francophone chez leur interlocuteur, ils étaient plus curieux que méprisants. Selon ces premiers, leur identité ethnique n’a posé aucun obstacle à leur intégration à la Floride. «Les gens voulaient savoir d'où tu viens, pourquoi t'es là, y'écoutaient notre accent. [...] Y'étaient curieux [...] Y'avait ben

42. Maurice Ouellette, «La Florida depuis 40 ans», Quelque part entre la 11 et la 17 (Sudbury, Première chaîne de Radio-Canada, $1^{\mathrm{er}}$ novembre 2006).

43. Yves Frenette, «La genèse de la communauté canadienne-française en NouvelleAngleterre: Lewiston (Maine), 1800-1880", Historical Papers/Communicatons historiques, 24, 1 (1989): 80-81.

44. Albert et Ginette [Deslauriers], Entrevue, Lake Worth (Floride), 7 janvier 2009. Ces derniers ont demandé que leur nom de famille soit substitué lorsque publié dans une étude.

45. Raymond Lemay, Entrevue, Lake Worth (Floride), 7 janvier 2009.

46. Denise D’Anjou, Entrevue, Lake Worth (Floride), 7 janvier 2009. 
du monde dans ce temps-là [durant les années 1960] qui ne savait pas qu’y avait du monde qui parlait [le] français au Canada ${ }^{47}$ !» Les immigrants canadiens-français acceptaient volontiers que l'anglais soit la langue d'usage en Floride. Selon l'historien Jean Lamarre, l'attitude par rapport à la langue est souvent dictée par la proportion d'une communauté immigrante à l'intérieur de sa localité d'adoption. À titre d'exemple, les Canadiens français de la vallée de la Saginaw (Michigan) étaient bien plus enclins à s'assimiler à l'anglais et à la naturalisation puisqu'ils ne composaient que $7 \%$ de la population locale, comparativement au 25 à $60 \%$ de francophones dans certaines localités de la Nouvelle-Angleterre ${ }^{48}$. La spécialité ouvrière aurait également contribué à l'accélération de leur intégration. Alors que le taux de naturalisation chez les Canadiens français était de $20 \%$ en Nouvelle-Angleterre et de $35 \%$ au Michigan en 1910, il était de $55 \%$ chez les Canadiens (français et anglais) en Floride en 1980. Par ailleurs, à Palm Beach, les résidants canadiens-français ne représentaient que $2 \%$ de la population totale du comté en $1970^{49}$. Le taux de naturalisation est relativement élevé lorsqu'on prend en compte que seulement $10 \%$ des résidants canadiens détenaient la citoyenneté américaine dans l'ensemble des États-Unis en $1990^{50}$. De plus, les Canadiens français provenaient du nord de l'Ontario, de la Nouvelle-Angleterre et de l'Estrie, soit des régions détenant des rapports considérables avec l'anglais. La forte présence de Yankees en Floride permettait également l'existence d'une culture locale moins étrangère que celle de l'Old South. Bureau éprouva des difficultés à s'adapter à un milieu "sudiste » ayant une «mentalité dure ${ }^{51}$ » dans le Nord floridien en 1964, mais elle fut réconfortée par la présence de Yankees et de Canadiens français lors de son arrivée à Palm Beach l’année suivante.

47. N. Bureau, Entrevue, op. cit., 9 janvier 2008.

48. Jean Lamarre, «L'intégration des migrants canadiens-français à la réalité socio-économique américaine : essai comparatif entre la Nouvelle-Angleterre et le Michigan ", dans Martin Paquêt et Andrée Courtemanche, dir., Prendre la route. L'expérience migrante en Europe et en Amérique du Nord du XIV au XXe siècle (Hull, Vents d'Ouest, 2001), 161.

49. «Florida + Palm Beach +1970 », Univerity of Virginia Library - Historical Census Browser, http: / / fisher.lib.virginia.edu/collections/stats/histcensus [site consulté le 21 juillet 2010].

50. «Table 195. Citizenship and Year of Immigration for Foreign Born Persons by Country of Birth: 1980 ", 1980 Census of Population. Volume I - Characteristics of the Population. Chapter D. Detailed Population Characteristics. Part 11 - Florida (Washington, US Bureau of the Census, 1983), 11/7. Nous n'avons pas trouvé de source nous permettant d'évaluer le taux de naturalisation chez les Canadiens français en 1980. R. Barde et al., "Chapter Ad - International Migrations... », op. cit., I-647.

51. N. Bureau, Entrevue, op. cit., 9 janvier 2008. 
La représentation de la Floride comme terra nullius est également pertinente dans la compréhension du comportement des Canadiens français. Marécageux et parsemé de fermes modestes, Palm Beach était peuplé par quelques Séminoles et Euro-Américains jusqu’en 1950. Doyon, travailleur en construction, n'y voyait que du potentiel au moment de son arrivée.

La construction, ça débutait! [...] C'était des fermes partout, c'était pas développé pantoute. [...] On était dans le bois. [...] La première maison que j’avais bâtie [faisait face à] des vaches juste sur le coin. [...] Y pompaient du sable pour faire ces quartiers ici ${ }^{52}$ !

Ouellette, quant à lui, louait des appartements à des Autochtones mayas du Guatemala qui se réfugiaient en Floride ${ }^{53}$. Une autre immigrante avouait qu'elle "détest[ait] assez les Cubains pour [s]'en confesser ${ }^{54}$ ». À son dire, son commerce de produits de beauté dut fermer ses portes à la suite de l'«invasion» cubaine de son quartier dans les mois suivant la révolution cubaine de 1959 . Un certain mépris des immigrants canadiensfrançais envers les autres nouveaux arrivants se manifestait par le fait qu'ils partageaient l'idée qu'il fallait «barr[er] [s]es portes ${ }^{55}$ » à la suite de l'arrivée (parfois illégale) de milliers de migrants latino-américains durant les années 1970 et 1980. Bien évidemment, l'impression d'être chez soi en Floride semble s'être développée rapidement...

Peu à peu, les réseaux de parents et d'amis devinrent des réseaux communautaires. Les immigrants canadiens-français se retrouvaient à la plage, dans les commerces et aux messes catholiques dites en anglais. Ils assistaient également à des événements sociaux où ils pouvaient converser en français. Il arrivait souvent aux Asselin de croiser d'autres immigrants canadiens-français dans les commerces de la ville. Après avoir entendu deux hommes blasphémer dans leur quincaillerie, les Asselin se présentèrent et une amitié s'est développé $e^{56}$. Palm Beach devint un pôle d'attraction de gens de l'Estrie, mais aussi des migrants du Nord de l'Ontario.

Prenons l'exemple de Ouellette pour illustrer comment un réseau migratoire peut se construire. En 1963, il visita un ami de Sudbury, Roger Bergeron, poseur de fenêtres qui gérait un motel à Palm Beach l'hiver. Sa conjointe, une Canadienne d'origine finlandaise, y avait été attirée par la

52. R. Doyon, Entrevue, op. cit., 8 janvier 2009.

53. Maurice Ouellette, Entrevue, Palm Springs (Floride), 7 janvier 2008.

54. [Identité dissimulée en raison de la controverse du commentaire], Entrevue, janvier 2008.

55. [Identité dissimulée], Entrevue, janvier 2008.

56. N. Bureau, Entrevue, op. cit., 12 janvier 2008. 
colonie de Finlandais de Lake Worth. Au cours de l'hiver, Bergeron fréquentait plusieurs Québécois. Ouellette côtoya ces derniers et fut persuadé que d'autres Canadiens français du Nord ontarien auraient envie de prendre des vacances dans ce nouvel îlot francophone. Dans les années qui suivirent, il encouragea ses beaux-parents, ses frères, ses sœurs et ses amis à séjourner à Palm Beach. Les connaissances de Ouellette ont aussi incité leurs proches à faire partie de la communauté. Ils renforçaient ainsi l'attrait de la région chez les Franco-Ontariens ${ }^{57}$.

Entre 1960 et 1970, le nombre de résidants canadiens-français de Palm Beach passa de 1700 à 3800 personnes $^{58}$. Ils composaient d'ailleurs entre 1 et $2 \%$ de la population totale, sans compter les milliers de touristes canadiens-français qui fréquentaient la région chaque année. Dès $1969^{59}$, le Beauceron Léo Pitt qui œuvrait en construction, invita des collègues canadiens-français et leurs familles chez lui où il animait des soirées sociales "pour se recréer et pour échanger sur leur présente situation ${ }^{60}$ ». À la suite du rassemblement de 80 Canadiens français, Pitt décida de louer une salle dès 1971 pour les réunions suivantes ${ }^{61}$. Il inaugura ainsi le premier club canadien-français de la région qui devint une association dûment constituée deux ans plus tard et qui prit pour nom le Club canadien-français d'Amérique (CCFA).

Les rencontres entre Canadiens français facilitaient la sociabilité tout autant que leur intégration au marché du travail et à la vie américaine. Selon le sociologue Raymond Breton, la complétude institutionnelle des communautés immigrantes ${ }^{62}$ vise aussi à «faciliter l'accès [aux] possibilités [de la majorité $]^{63}$ ». Yves Roby abonde également dans ce sens. Pour lui, la paroisse, l'école, la mutuelle et la presse déployaient à la fois des efforts

57. M. Ouellette, Entrevue, op. cit., 5 janvier 2009.

58. La méthodologie des calculs précédents pour quantifier la proportion grandissante des Canadiens français par rapport à la population totale est réutilisée ici. «Florida + Palm Beach + Canadian + French Canadian + 1960 », Univerity of Virginia Library - Historical Census Browser, http: / / fisher.lib.virginia.edu/collections/stats / histcensus [site consulté le 21 juillet 2010]; "1950 $+1960+1970+1980+1990+$ Social and Economic Characteristics + Florida», Census of Population and Housing - Decennial Censuses, www.census.gov/prod/www/abs/decennial/ index.htm [site consulté le 21 juillet 2010].

59. Maurice Ouellette, "Maurice Ouellette», dans Thérèse St-Amour, dir., Récits de la Floride (Delray Beach, The Printing Office, 2006), 79.

60. "Léo Pitt et le Club canadien-français», dans T. St-Amour, ibid., 75.

61. R. Brousseau, Entrevue, op. cit., 12 janvier 2008.

62. Raymond Breton, «Institutional Completeness of Ethnic Communities and the Personal Relations of Immigrants », American Journal of Sociology, 70, 2 (septembre 1964): 193-205.

63. Raymond Breton, "L’intégration des francophones hors Québec dans des communautés de langue française », Revue de l'Université d'Ottawa, 55, 2 (avril-juin 1985): 78. 
pour faire germer une vie française tout en encourageant un enracinement dans la société d'accueil. Ces objectifs en apparence contradictoires ont coexisté pendant quelques décennies en Nouvelle-Angleterre, avant de largement céder au deuxième objectif durant l'entre-deux-guerres et définitivement après la Deuxième Guerre mondiale ${ }^{64}$.

Selon ses fondateurs, le CCFA avait pour but de «réunir la famille», «les Canadiens» et «les familles canadiennes ${ }^{65}$ » à Palm Beach ou même les Canadiens français "d'Amérique», faisant allusion à leur présence aux États-Unis. Les règlements administratifs de 1973 en soulignent les visées économiques et sociales.

The corporation is organized and chartered expressly for a means of promoting the social and welfare interests and good will of French Canadian residents, their friends and French Canadian visitors in the State of Florida and for the purpose of providing management, consulting and advisory services to its members, upon a fee basis or otherwise $e^{66}$.

L'organisme aspirait donc à veiller au bien-être des Canadiens français immigrants d'abord, puis de leurs amis (voisins et autres immigrants non canadiens-français) et, en dernier lieu, des visiteurs canadiens-français. Le CCFA se dota d'un conseil d'administration (CA), d'un conseil de direction (CD) et tint des assemblées générales annuelles. Le CCFA eut sept présidents entre 1973 et $1982^{67}$ et sa charte fut révoquée en 1977 lorsque la Florida Division of Corporations constata qu'elle n'avait pas reçu de rapport annuel en $1976^{68}$. Malgré tout, l'organisme continua d'exister.

Les rapports d'activités de 1973-1974 font état de soirées dansantes aux airs traditionnels du violon du président Pitt qui s'organisent tous les deux samedis $^{69}$. Comme le confirme un album souvenir d'une soirée de l'automne 1976, la moitié des participants étaient des résidants de la Floride alors que l'autre moitié comptait des résidants ontariens et québécois. Quelques couples provenaient de la Nouvelle-Angleterre. En tout, 84\%

64. Yves Roby, Les Franco-Américains de la Nouvelle-Angleterre, 1776-1930 (Sillery, Septentrion, 1990).

65. S. Doyon, Entrevue, op. cit., 8 janvier 2009.

66. Certificate of Incorporation of Club canadien-français d'Amérique Incorporated, 30 août 1973, 4, dans: Fonds Division of Corporations (NP 27 325), Tallahassee (Floride), Florida Department of State Archives (FDSA).

67. "Ordre chronologique des présidents du Club canadien-français», dans T. St-Amour, Récits de la Floride, op. cit., 74-75.

68. Ibid., i, 3-8; "Club Canadien-Francais d'Amerique Inc.», Division of Corporations Archives, dans: FDSA, www.sunbiz.org [page consultée le 21 juillet 2010].

69. "Léo Pitt et le Club canadien-français», T. St-Amour, Récits de la Floride, op. cit., 76; Armand Lallinec, Entrevue, Lake Worth (Floride), 10 janvier 2008; C. Roy, Entrevue, op. cit., 8 janvier 2008. 
des participants possédaient des noms de famille canadiens-français ${ }^{70}$, un signe de la capacité de l'organisme d'attirer des gens d'un peu partout dans le Nord, tout en accueillant des concitoyens de groupes culturels variés. Raymond Breton appellerait ces non canadiens-français le «marché extérieur» de l'organisme ${ }^{71}$. Les nombreuses photographies suggèrent un enracinement et un attachement affectif au milieu d'accueil avec la présence simultanée des drapeaux américain et canadien autant dans les documents d'archives que dans les formulaires et les publicités du CCFA ${ }^{72}$.

Le président en 1976-1977, Conrad Roy, promouvait l'organisme lors de séjours de recrutement au Québec ${ }^{73}$, mais, selon lui, il aurait été mal reçu par plusieurs personnes approchées ${ }^{74}$. Il se peut que l'idée de l'exode aux États-Unis ait fait vibrer des cordes sensibles dans la Belle Province. Certains nouveaux arrivants parlant peu anglais, Roy servit également d'intermédiaire ethnique. Toujours fidèle à sa volonté de bâtir des liens avec d'autres groupes et des Floridiens américains, comme le révèlent les documents de fondation et les entrevues, le CCFA commémora le bicentenaire de la Révolution américaine le 3 avril 1976 à West Palm Beach. Des membres des communautés ukrainienne, polonaise, tchèque, slovaque et finlandaise de la région participèrent à ce «United Nation». On y présenta plusieurs numéros débutant avec le chant du Star Spangled Banner par l'immigrant acadien Ovila Cormier suivi par l'ô Canada. Quatre couples dansèrent sur des airs folkloriques tels que Vive la Canadienne et Alouette. Dans l'album souvenir, on pouvait lire que «nous Canadiens français sommes fiers de représenter notre pays le Canada par nos chants, notre musique et nos danses ici aux États-Unis à West Palm Beach $^{75}$ ». À l'issue de la soirée, 156 personnes signèrent cet album. Soixante-sept d'entre elles (43\%) avaient des noms canadiens-français. Les participants ayant une résidence permanente ailleurs qu'en Floride provenaient du Québec, de l'Ontario, de l'Ohio, du Massachusetts et de New York $^{76}$.

70. «Première danse d'automne», novembre 1976, Club canadien-français de Lake Worth..., op. cit.

71. R. Breton, «L’intégration des francophones hors Québec... », loc. cit., 87.

72. "St-Valentin 1976», Club canadien-français de Lake Worth..., op. cit., 1.

73. "Club canadien-français d'Amérique en Floride », La Presse, [ca. 1977]; "Going to Florida?», [journal anglophone inconnu], [ca. 1977], Club canadien-français de Lake Worth..., op. cit.

74. Roy, Entrevue, op. cit., 8 janvier 2008.

75. Musique, chants et danses interprétées par les Canadiens à West Palm Beach, Florida, 3 avril 1976, dans: Archives personnelles de Simone Doyon (APSD), Palm Springs (Floride).

76. "United Nation", Club canadien-français de Lake Worth..., op. cit. 
Tous les deux dimanches, on proposait un programme d'activités aux enfants des immigrants. En décembre 1978, le Palm Beach Post s'entretenait avec Simone Doyon qui était heureuse que ses enfants pêchent et cueillent des baies sauvages avec leur grand-père au Québec. Elle se disait fière que ses enfants nés en Floride maîtrisent le français et l'anglais. Les activités du CCFA aidaient aussi à maintenir la langue vivante chez les enfants. «In fact, mentionnait Doyon, all the French club members['] children can understand it, even if they can't speak it ${ }^{77}$."

Comme toute autre îlot canadien-français, celui de Palm Beach a dû se pencher sur la langue principale par laquelle elle communiquerait. En Nouvelle-Angleterre, dès la fin du XIX ${ }^{\mathrm{e}}$ siècle, des curés de paroisse abandonnaient leur liturgie et leur instruction unilingue au profit d'un bilinguisme intégral. Plusieurs furent même persuadés que le bilinguisme institutionnel serait plus apte à maintenir les jeunes Franco-Américains dans la communauté et à répondre à leurs besoins dans un contexte étatsunien $^{78}$. Près d'un siècle plus tard, les dirigeants de la communauté canadienne-française de Palm Beach ne cherchaient plus à maintenir les liens avec la patrie par l'entremise de la langue et de la foi. Bien que le français ait été incontestablement une langue de sociabilité, Roy croyait que l'anglais devait aussi avoir sa place au CCFA. Selon lui, l'organisme devait être bilingue pour faciliter l'intégration des immigrants canadiensfrançais. Une attitude hostile à l'anglais ne pourrait que menacer l'harmonie avec leurs concitoyens non francophones. «Y'a tout le temps [eu] de l'anglais dans le Club. [...] La moitié [des membres] étaient des Américains $^{79}$. [...] Au commencement, on prenait n'importe qui quand y voulaient venir. [...] Y'en avaient gros qui parlaient [uniquement l']anglais ${ }^{80} . »$

Breton note par ailleurs que ce type de rassemblement peut permettre la formation d'un réseau extracommunautaire. La présence d'Américains anglophones aux activités serait donc cruciale. Roy notait le mal qu'éprouvaient plusieurs Canadiens français à communiquer et il était conscient de l'avantage que son bilinguisme lui procurait. Il lui permettait de travailler, mais aussi de poursuivre l'American Dream. Roy ne se voulait pas un agent d'assimilation puisqu'il tenait à communiquer avec ses enfants en français, mais il la considérait comme une langue familiale bien plus

77. Nancy Figel, «Simone Doyon displays some of her French Canadian craft work», The Palm Beach Times (12 décembre 1978), dans: Fonds Populations (Canadians in Florida), HSPBCA.

78. Y. Roby, Les Franco-Américains de la Nouvelle-Angleterre..., op. cit., 188-191.

79. Il est probable qu'il se réfère aux Franco-Américains, mais n'avons pas pu le confirmer.

80. C. Roy, Entrevue, op. cit., 8 janvier 2008. 
qu'une langue publique. Alors que les enfants pouvaient perfectionner leur anglais à l'école, les adultes devaient être en mesure de le faire dans leur communauté.

Parce que t'es en Amérique, tu commences pas à parler français. Tu parles en anglais pour commencer puis après tu parles [en français]. Y'en a qui disaient «Ahhhh, y s'en devient Américain!» Le problème c'est qu’y voulaient que je parle en français [seulement], mais on avait autant d'Anglais ${ }^{81}$ dans le club. [...] On les prenait comme membres même si y étaient pas Canadiens ${ }^{82}$.

Sa vision se confrontera à celle d'une partie du groupe qui le chassa de la présidence en 1977. "Ah oui, je me suis fait haïr», confiait-il. De plus, un nombre grandissant des participants étaient des hivernants. L'intégration linguistique que proposait Roy n'était peut-être pas nécessaire non plus puisque plusieurs immigrants étaient bilingues au moment de leur arrivée en Floride. Il est donc possible que plusieurs se soient questionnés sur la pertinence de l'organisme.

\section{L'ESPRIT NATIONALITAIRE DES HIVERNANTS EN FLORIDE, I 977 - I 997}

Si la résistance à l'intégration économique de l'Amérique du Nord a caractérisé les politiques commerciales du Canada jusqu’à la Deuxième Guerre mondiale, la géopolitique de l'après-guerre privilégia la régionalisation des échanges en Europe comme en Amérique du Nord. La diminution des transactions avec la Grande-Bretagne au profit du commerce canado-américain amena la conclusion d'ententes de libre-échange concernant des denrées spécifiques tel que le pacte de l'auto de $1965^{83}$. Malgré leur rhétorique antiaméricaine, les gouvernements libéraux et conservateurs poursuivirent le développement des liens économiques continentaux et préparèrent le terrain pour l'adoption de l'Accord de libre-échange entre le Canada et les États-Unis en $1988^{84}$. Loin d'y voir une abdication de souveraineté, l'historien Bruce Muirhead en discerne plutôt un choix pragmatique permettant au Canada de connaittre une prospérité inégalée.

81. Nous ne savons pas s'il entendait des Américains, des Canadiens anglais ou des gens bilingues.

82. C. Roy, Entrevue, op. cit., 8 janvier 2008.

83. Bruce Muirhead, Dancing Around the Elephant: Creating a Prosperous Canada in an Era of American Dominance (Toronto, University of Toronto Press, 2007).

84. Il devint l'Accord de libre-échange nord-américain à la suite de l'ajout du Mexique au pacte en 1993. 
Par ailleurs, plusieurs entreprises canadiennes rencontrèrent un succès autrefois impensable aux États-Unis et en Europe. Ainsi, l'implantation de Bombardier en Europe et aux États-Unis tout comme l'acquisition d'entreprises américaines par Quebecor poussèrent des analystes à baptiser le phénomène "Québec Inc. ${ }^{85}$ ". De plus, l'investissement canadien à l'étranger s'est multiplié par 17 entre 1960 et 1984 et a atteint 41 milliards de dollars ${ }^{86}$. Les Canadiens possédaient de 12 à $15 \%$ de toute propriété étrangère en Floride et formaient le groupe d'investisseurs étrangers le plus important de l'État en $1978^{87}$. Durant ces années, une affluence de travailleurs canadiens se trouvait au sud $\mathrm{du} 45^{\mathrm{e}}$ parallèle pendant des périodes prolongées sans y prendre une résidence permanente pour autant. Au-delà du libre-échange croissant, certains journalistes pointaient vers l'incertitude politique créée par l'élection du Parti québécois en novembre 1976 pour expliquer la hausse des acquisitions en Floride.

Les dernières années de la décennie 1970 se sont avérées charnières en Floride. Au Québec, le gouvernement du Parti québécois se vouait plus que jamais à la protection du fait français (par l'adoption de la Loi 101 entre autres), se faisait promoteur de l'indépendance du Québec et augmentait ainsi la ferveur nationaliste des francophones du continent. Pour sa part, le CCFA se choisit un président plus nationaliste désirant se rapprocher plus des hivernants canadiens-français que des autres groupes ethniques. C'est aussi à cette époque que les hivernants deviennent à peu près aussi nombreux que les immigrants en Floride.

Afin de comprendre le phénomène des hivernants, il faut remonter à la genèse des régimes de retraite, régimes qui permirent à un nouveau segment de la population de rêver à une retraite plus active, notamment grâce aux avancées médicales qui prolongeaient l'espérance de vie. L'historien Gary Mormino constate d'ailleurs que ces régimes ont permis la démocratisation du tourisme prolongé au troisième âge ${ }^{88}$. Dès 1951 , Ottawa rendit la retraite obligatoire pour toutes les personnes ayant plus de 70 ans et étendit le régime de "pension du Canada ${ }^{89}$ " à tous ses citoyens âgés. Cette mesure permettait aux aînés de demeurer des consommateurs actifs. Durant les années 1960, l'augmentation de l'espérance de vie et les

85. Robert Chodos et Eric Hamovitch, Quebec and the American Dream (Toronto, Between the Lines, 1991), 195-200.

86. P.-A. Linteau, Histoire du Québec contemporain..., op. cit., 450-451.

87. Jody Long, "Canadian Investment Piling Up: Backers Like Business Climate Here», The Palm Beach Post, 19 novembre 1978, FF1-FF2, dans: Fonds Populations (Canadians in Florida), HSPBCA.

88. G. Mormino, Land of Sunshine, State of Dreams..., op. cit., 128.

89. L'âge minimal sera ramené à 65 ans quelques années plus tard. 
pensions de retraite firent des retraités un groupe démographique influent ${ }^{90}$. Alors qu'auparavant les Canadiens français s'installaient en Floride pour améliorer leur sort, les touristes et les hivernants s'y permettaient des vacances grâce à leur nouvelle aisance matérielle. Les campagnes de promotion de l'État signalaient à quel point il était possible de vivre et investir en Floride même à partir de revenus modestes ${ }^{91}$. Malgré la tentation d'adapter un style de vie nomade, les Canadiens du troisième âge maintenaient un port d'attache au pays surtout pour ne pas perdre l'accès au régime de santé publique qu'une absence de six mois consécutifs de leur province de résidence leur aurait enlevée $e^{92}$.

Alors que le nombre de résidants canadiens passait de 58000 à $155000^{93}$ entre 1960 et 1990 (une augmentation de $300 \%$ ), le nombre de touristes canadiens (y compris les hivernants) passait de $245000^{94}$ à $2400000^{95}$ pendant la même période (une augmentation de $1000 \%$ ). En 1982, les migrations des hivernants avaient pris une ampleur inouie au point qu'elles suscitèrent la remarque suivante du Florida Department of State: "Florida is appreciated for being "just like Toronto/Montreal but with sun" in the wintertime. [...] The concept that "Florida is American" [is] an advantage [that] manifests itself in terms of familiarity with the culture, food and language ${ }^{96} . »$ Kathy English du Toronto Star écrivait en 1986: "Florida is no longer the Canadian dream vacation - just a realistic one. We leaf through travel brochures looking for the exotic, knowing in the

90. P.-A. Linteau, Histoire du Québec contemporain..., op. cit., 327-330, 444.

91. G. Mormino, Land of Sunshine, State of Dreams..., op. cit., 132.

92. Les lois ontariennes et québécoises sont très semblables, sauf qu’un résidant québécois peut maintenir son statut de résidence à condition que son absence allant jusqu'à un an ne se produise qu'une fois aux sept ans; David Reeves Counts et Dorothy Ayers Counts, Over the Next Hill. An Ethnography of RVing Seniors in North America (Peterborough, Broadview Press, 2001), 301, $303,308$.

93. «1960 + $1990+$ Social and Economic Characteristics + Florida», dans: Census of Population and Housing - Decennial Censuses, www.census.gov/prod/www/abs/decennial/ index.htm [site consulté le 21 juillet 2010].

94. G. Desrosiers-Lauzon, "À l'envers de l'hiver... », loc. cit., 112.

95. E. Jarvis, «Florida’s Forgotten Ethnic Culture... », loc. cit., 186-197; Sylvain de Repentigny, International Travel Survey: Canadian Residents 1980 - 1999. Data Selected: U.S. State Visits, Including En Route. Total U.S. State Visits Selected, U.S. Regions by U.S. States, $1+$ Nights by Visits and Spending Less Fares. Florida 1980-1999, Gatineau, Statistique Canada, texte inédit, 30 juillet 2007, 1.

96. James P. King et David A. Lyon, Canadian Travel Patterns and Attitudes Towards Vacations in Florida (Toronto/Tallahassee, Market Facts of Canada/Florida Department of State/Florida Department of Commerce, 1982), 40. 
back of our minds that "we can always go to Florida" ". " La destination s'intégra à l'imaginaire canadien au point d'en devenir une forme de prolongement.

La Floride des années 1970 subissait des vagues migratoires qui la transformèrent, à la fois, en paradis de retraités et en refuge pour de nombreux Latino-Américains. Alors que ces derniers s'enracinèrent en Floride en raison du maintien du régime castriste à Cuba, les Canadiens cessèrent d'émigrer en Floride (ou à peu de chose près) au profit d'une nouvelle habitude qui fit de nombreux adeptes chez les retraités. Déjà en 1984, les gérontologues Larry Tucker et Charles Longino recensaient environ 250000 hivernants canadiens en Floride ${ }^{98}$ qui y séjournaient de un à six mois par année. Il est probable que 100000 d'entre eux aient été canadiens-français ${ }^{99}$. En 1987, le Conference Board of Canada estimait que ce nombre s'élevait à $300000^{100}$.

Les hivernants se distinguent des immigrants en ce qu'ils n'adoptent ni une citoyenneté nouvelle ni un statut permanent. Ils sont souvent à la retraite et d'âge mûr. Ils s'intègrent à une communauté d'accueil à un degré impressionnant, mais ils s'intéressent rarement à la société locale. Les référents sociaux tout comme leurs regards s'orientent donc davantage vers le Canada ou ses ressortissants et se détournent de la Floride. Dans ce cas, explique Rosental, la transplantation est «secondaire dans la mesure où ses acteurs maintiennent fondamentalement $[\ldots]$ le même cadre de vie, que leurs compatriotes restés sur place ${ }^{101} »$. Ils ne sont pas non plus des touristes puisqu'ils s'investissent dans leur communauté et adoptent un rythme de vie plus routinier. Ainsi, les Canadiens français en vinrent à partager leur temps entre deux milieux de vie. L'émigration n'était plus obligatoire pour résider en Floride l'hiver que ce soit pour

97. Kathy English, «Florida prospers on Canadian dollars. Exchange rate makes vacations or residences more expensive but millions of Canadians flock to the Sunshine State», Toronto Star, 9 mars 1986, A12.

98. G. Desrosiers-Lauzon, «À l'envers de l'hiver... », loc. cit., 113.

99. Hollywood, à lui seul, recevait 60000 hivernants québécois francophones en 1982 et Tucker estime que 35 à $40 \%$ de la population hivernante canadienn était francophone en 1988 ; Louis Dupont et Marie Dussault, «La présence francophone en Floride : un portrait », Vie française, 36 (octobre-décembre 1982), 5-22; Richard D. Tucker, Larry C. Mullins, Charles F. Longino Jr., Victor W. Marshall et François Béland, «Older Canadians in Florida: A Comparison of Anglophone and Francophone Seasonal Migrants", La revue canadienne du vieillissement, 11, 3 (automne 1992): 281-299.

100. Jon Nordheimer, «Hockey draws "two solitudes" together», The Windsor Star, 9 avril 1987, B8; Michael MacKenzie, Canadian Snowbird Association, Entretien téléphonique, Toronto, 15 novembre 2008.

101. P.-A. Rosental, «Maintien/rupture...», op. cit., 1410. 
deux semaines ou pour l'hiver entier. Il devint conséquemment de moins en moins nécessaire de quitter son espace investi (le Canada français) vu le niveau d'organisation communautaire en Floride.

Au-delà des 9100 résidants d'origine canadienne-française de Palm Beach, on aurait compté environ 7000 hivernants canadiens-français ${ }^{102}$ en 1990. En plus d'être aussi nombreux, ces derniers étaient proportionnellement plus enclins à participer à la vie communautaire que les immigrants qui détenaient des emplois et qui s'étaient intégrés depuis des années. L'arrivée annuelle d'une masse de retraités eut des répercussions appréciables sur le Club canadien-français d'Amérique. Il privilégia, peu à peu, le maintien des liens avec le Canada français et la cohésion sociale de ses citoyens au détriment de l'intégration à la société américaine. Le CCFA s'est donc lentement réorienté vers une clientèle presque exclusivement francophone. Lorsque Roland Doyon accéda à la présidence en 1977, au lieu d'intégrer de nouveaux immigrants (au Québec et en Floride) pour assurer la stabilité de l'organisme comme Roy l'avait suggéré, il sollicita la participation des hivernants canadiens-français à Palm Beach. Le taux de non-francophones participant aux événements diminua, ce qui fit écho à ce changement de $\operatorname{cap}^{103}$.

Durant la soirée du 13 janvier 1979, Doyon souhaita la «bienvenue à tous du Québec et Ontario » en demandant aux hivernants "de s'unir à nous les membres». Sa volonté d'employer les termes "union» ou "unir» au cours de nombreux rassemblements semble suggérer une solidarité renouvelée entre Canadiens français, quel que soit leur lieu d'origine ou leur statut de résidence. Étant entrepreneur, il est possible que Doyon ait pensé qu'il serait plus profitable de recruter des hivernants canadiensfrançais que d'interpeller la participation des autres communautés ethniques. Le bilinguisme fut progressivement abandonné et les soirées dansantes mirent désormais l'accent sur la musique "canadienne» ${ }^{104}$. Il est bien possible que plus de la moitié des participants au dîner du 10 mars 1979 ait été des hivernants: la quasi-totalité des 43 invités était des hiver-

102. L'estimation de 100000 et 120000 hivernants canadiens-français est la statistique croisée avec celle qui veut que $6 \%$ de la population canadienne-française de la Floride se trouve à Palm Beach en 1988. La population aurait donc atteint entre 6000 et 7200 hivernants canadiens-français durant cette période. R. Tucker et al., "Older Canadians in Florida... », op. cit., 281-299.

103. «Soirée d'ouverture nov 1977 », «Jour de l’An 1978», «Jan. 14 1978», «Janv. 28 1978», «Feb11-78», «Feb-18-78», "Mars 11 1978», "May 1978», "Juin 1978», «Nov 25 1978», «Dec $1678 »$, Club canadien-français de Lake Worth..., op. cit.

104. «Jan 13 1979»; "Jan 27 1979»; «Feb 10 1979»; Club canadien-français de Lake Worth..., op. cit. 
nants et les 86 membres étaient divisés entre les hivernants et les immigrants ${ }^{105}$.

Toutefois, cette nouvelle solidarité ne fit pas l'affaire de certains immigrants canadiens-français qui délaissèrent l'organisme par "manque d'intérêt». À la suite de l'établissement de réseaux personnels et professionnels (et donc de l'atteinte du but fixé au moment de la fondation du CCFA), l'organisme devenait inutile pour certains. «On s'est fait des amis, on a[vait] de la famille et on s'intéressait moins à ça ${ }^{106}$ ", expliquait Bureau. Le fait que l'immigration canadienne en Floride et aux États-Unis ait chuté dramatiquement au lendemain de l'adoption du Preference System réduisit la nécessité d'un club orienté vers les immigrants ${ }^{107}$. De plus, le CCFA devint déficitaire, car il recruta en deçà de la masse critique nécessaire à sa rentabilisation. Des conflits de personnalités et de la corruption auraient aussi été à la source des difficultés ${ }^{108}$. Enfin, la présence accrue d'hivernants franco-ontariens aurait également créé des tensions; certains immigrants québécois se voyant contester la direction sur deux fronts. En 1981, les activités du CCFA furent interrompues pendant un an, faute de programmation et en raison du décès de son président.

La direction du CCFA fut reprise par des hivernants. Ayant quitté la présidence depuis quelques années, Doyon considérait que les hivernants étaient allés trop loin dans l'adaptation de l'organisme. Les hivernants, se plaignait-il, «nommaient leurs membres, votaient pour eux[-mêmes]» et devinrent "plus forts que nous autres ${ }^{109}$ ". En 1980, Ouellette devint le premier hivernant membre de l'exécutif du CCFA et son premier président hivernant en 1982. La tradition fut maintenue pendant le quart de siècle qui suivit, car tous les présidents ont été hivernants et FrancoOntariens, ce qui fait quand même écho aux frustrations de Doyon. Homme d'affaires, Ouellette ne conçut plus le CCFA comme un organisme d'intégration à la majorité ni comme un organisme orienté vers les besoins des immigrants. Il mit fin aux activités d'été et aux activités familiales. Il chercha à rentabiliser le CCFA en lui donnant un cachet culturel particulier afin qu'il puisse occuper son propre édifice. Par ailleurs, il insista que les activités soient bien fréquentées et que les Franco-Ontariens

105. «March 10 1979», Club canadien-français de Lake Worth..., op. cit.

106. N. Bureau, Entrevue, op. cit., 9 janvier 2008.

107. R. Barde et al., "Chapter Ad - International Migrations», loc. cit., I-571-I-572.

108. R. Brousseau, Entrevue, op. cit., 12 janvier 2008; C. Roy, Entrevue, op. cit., 8 janvier 2008; Armand Lallinec, Entrevue, Lake Worth (Floride), 10 janvier 2008.

109. R. Doyon, Entrevue, op. cit., 8 janvier 2009. 
soient représentés au CA. Pour ce faire, il instaura une nouvelle composition du Conseil d'administration dans le but de mieux refléter les lieux d'origine de la population canadienne-française.

En 1980, le groupe était surtout formé de Québécois. Durant les premières années du Club, les gens se chicanaient et se volaient. J’ai fait que mon conseil [ait] des Américains et des Ontariens. Je m'assurais que notre conseil [ait] quatre Ontariens, deux Américains et deux Québécois. De même, on n’allait pas faire de jalousie. Et ça allait bien ${ }^{110} \ldots$

Le fait que tous les élus soient des hivernants ou que les FrancoOntariens occupent la moitié des sièges du CA ne semble pas tenir compte de la réalité démographique de la communauté non plus. Si un doute plane sur la véritable représentativité de la nouvelle structure du CA, la solidarité nouvelle s'exprimait d'abord et avant tout par son succès à recruter des participants. Le discours de Ouellette n'était pas en contradiction avec celui de Doyon par rapport à l'unité recherchée entre Canadiens français en Floride, même si Ouellette privilégiait manifestement les hivernants. Pour eux, il fallait consolider la communauté avant d'y inviter des gens provenant d'autres groupes ethniques. Et l'unité recherchée n'était pas acquise: les Franco-Ontariens avaient tendance à fréquenter le Polish Club local au lieu du CCFA dans les années $1970^{111}$. L'effort de consolidation provoqua une expression de solidarité intéressante, pourtant absente du discours des fondateurs. Roby note d'ailleurs que la vague de nouveaux migrants canadiens-français arrivés durant les années 1920 en Nouvelle-Angleterre avait tendu les relations communautaires en ranimant une ferveur nationaliste au sein des organismes existants $^{112}$.

Ouellette fit la promotion de l'organisation auprès des gens qui «sont arrivés hier ${ }^{113}$ » uniquement en français. Lorsqu'il exprimait son désir de recruter des "Canadiens», il semble faire référence aux Canadiens français dans leur appellation historique du XVIII ${ }^{e}$ et du XIX ${ }^{e}$ siècle. Il sollicita également la participation de Français et d'Américains francophones. Son recrutement fut donc à forte saveur ethnique sans toutefois être sélectif. Au début des années 1980, Ouellette plaçait des pancartes sur la plage pour que les «Canadiens» prennent conscience de l'existence du CCFA 
et qu'ils y participent. "Quand j’entendais parler français dans les maga[s] ins et sur la rue, je leur disais qu[']on avait un club canadien et [je] leur demandais de venir se joindre à nous ${ }^{114}$.» En offrant "une danse et une drink gratis ${ }^{115}$ " à ses membres, il tâchait de fidéliser sa clientèle.

Sa réorientation connut le succès : durant la saison 1984-1985, l'association comptait plus de 150 membres en plus d'un nombre plus important de participants ${ }^{116}$. À la suite de la location de la salle Casino Hall qui donne sur l'Atlantique à Lake Worth, le CCFA n'éprouvait plus de difficulté à remplir la salle de 293 personnes à sa pleine capacité. Les grands bazars, les pique-niques, les soirées de réveillon étaient aussi au programme durant les années 1980 et $1990^{117}$. La coordonnatrice des repas, Jeannette Dalcourt de Verner (Ontario), préparait «jusqu’à 40 tourtières, 2000 boulettes, 2 fesses de cochon, puis 7 dindes " à l'aide de son équipe de cuisinières ${ }^{118}$. En peu de temps, l'organisme devint même rentable ${ }^{119}$. À titre d'exemple, entre les automnes 1993 et 1994, les acquis financiers du CCFA passèrent de 18500 à $54200 \$$. La somme de $39200 \$$ comptants semble même avoir été suffisante pour faire un premier versement en vue de la construction d'un édifice ${ }^{120}$.

Les Franco-Ontariens auraient pu s'installer avec leurs concitoyens ontariens sur la côte ouest de la Floride et auraient pu aussi participer au Canadian Club of the Palm Beaches (CCPB) composé à majorité d'Ontariens anglophones. Pourtant, ils optèrent à la longue pour des organismes canadiens-français, ce qui semble confirmer la volonté de maintenir leur langue et culture d'origine pendant leur séjour floridien. Ouellette

114. Maurice Ouellette, Le Club Canadien de Lake Worth, Floride, 2003, dans : ACCFLW.

115. M. Ouellette, Entretien téléphonique..., op. cit., 25 octobre 2006.

116. Jeannette Dalcourt, Entrevue, Greenacres (Floride), 7 janvier 2008; M. Ouellette, Entretien téléphonique, op. cit., 25 octobre 2006.

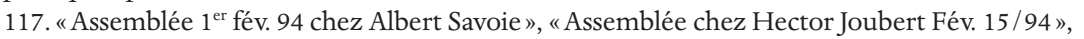
"Assemblée Mars $1^{\text {er }} 94$ chez Georgette Gamache», "Assemblée 17 Jan 95 Chez Georgette Gamache», "Assemblée Mars le 13/95 chez Georgette Gamache», "Assemblée 5 Fév 97 chez Hector Joubert», "Assemblée régulière du 19 mars 1997 chez M. Joubert», Club canadien-français d'Amérique/ de Lake Worth 1994-2005, 2, 6-8, 12, 18, 33; dans: ACCFLW.

118. J. Dalcourt, Entrevue, op. cit., 7 janvier 2008.

119. M. Ouellette, Entrevue, op. cit., 7 janvier 2008.

120. First Federal of the Palm Beaches Statement of Account Transactions - Club Canadian Francais Six Month Certificate 114008007, West Palm Beach, 29 mars 1991, 28 juin 1991, 30 septembre 1991, 31 décembre 1991, 30 juin 1992, 31 décembre 1992, 31 mars 1993, 30 juin 1993, 30 septembre 1993, 31 décembre 1993, 30 juin 1995, 11 p.; First Federal of the Palm Beaches Statement of Account Transactions - Club Canadian Francais One Year Certificate 1144022358, West Palm Beach, 30 juin 1992, 30 septembre 1992, 31 mars 1993, 30 juin 1993, 31 décembre 1993, 31 mars 1994, 30 juin 1995, dans: ACCFLW. 
constata que le CCFA n'avait pas établi de lien avec le CCPB durant ses années à la présidence, car "c'est pas le même monde, y’ont pas nos habitudes. Y vont pas venir danser à des chansons françaises! [...] Y'aiment jouer aux cartes eux autres, puis c'est ben correct, mais c'est pas pour moi ${ }^{121}$. Il paraît que même les hivernants canadiens-français du Vermont préféraient s'entretenir avec des «Canadiens» qu'avec leurs voisins anglophones du Vermont ${ }^{122}$. Dalcourt poursuivait:

Les gens [étaient] contents de rencontrer quelqu'un d'ailleurs, qu'y viennent du Nouveau-Brunswick ou qu'y viennent d'où y viendront je trouv[ais] que les gens [étaient] toujours voulant [sic] de rencontrer les autres, de faire des nouveaux amis puis de parler de leurs problèmes. Je ne trouve pas qu' [il existait] une friction [entre Canadiens français de différents lieux d'origine]. J'trouv[ais] que les gens [étaient] plutôt unis. [...] Nous autres, on a[vait] toutes sortes de monde, puis j'pens[ais] que plus gros [était] la famille, mieux c'[était]! [...] Y'en a[vait] des Québécois qui p[ouvaient] être piquants des fois, mais j'sais pas, pas au point de s'haïr, de se rivaliser. Moi, je trouv[ais] que $c^{\prime}[\text { était] moins pire [qu’à Floribec] }]^{123}$.

Selon Dalcourt, Floribec aurait été composé de Montréalais qui formaient une communauté "serrée » peu encline à s'ouvrir aux gens d'autres milieux d'origine. Le sondage de cette recherche révélait d’ailleurs une grande cohésion entre Franco-Ontariens et Québécois à Palm Beach, notant seulement quelques distinctions perceptibles entre ces "Canadiens». Les Québécois semblaient qualifier leurs relations avec les Franco-Ontariens d' "excellentes", alors que les Franco-Ontariens avaient plus tendance à les trouver "bonnes ${ }^{124}$. Malgré ses défauts, la composition du CA représentait les différentes régions du Canada français. Les attitudes des uns envers les autres semblent nuancer un ressentiment censé être omniprésent entre les francophones en milieu minoritaire et les Québécois francophones à partir des années 1960. Les quelques embêtements découlaient surtout de la méconnaissance des Québécois de la réalité francophone en milieu minoritaire au Canada. Le sondage révélait pourtant qu'ils choisissaient de s'identifier comme "Canadiens» ou "Canadiens français», un autre élément leur permettant de faire une

121. M. Ouellette, Entrevue, op. cit., 7 janvier 2008.

122. R. Lemay, Entrevue, op. cit., 7 janvier 2009; A. Savoie, Entrevue, op. cit., 7 janvier 2009; A. et G. [Deslauriers], Entrevue, op. cit., 7 janvier 2009; Carmen Bernier, Entrevue, op. cit., Palm Springs (Floride), 5 janvier 2009.

123. J. Dalcourt, Entrevue, op. cit., 7 janvier 2008.

124. Sondage auprès des Canadiens français de Palm Beach, janvier 2008. 
communauté en Floride ${ }^{125}$. Leur comportement rejoint précisément ce qu'avance Harney sur le regroupement culturel, national ou ethnique de gens en Floride nonobstant les origines territoriales distinctes ${ }^{126}$. Cette solidarité à Palm Beach semble nuancer les conclusions des historiens Gaétan Gervais, Yves Frenette et Marcel Martel qui caractérisent d'irréconciliable la division entre le Québec français et les minorités de la diaspora après la Révolution tranquille ${ }^{127}$. Si l'idée du Canada français s'est largement affaiblie, elle semble profiter d'une forme de "respect ${ }^{128}$ ", comme le diraient E.-Martin Meunier et Joseph Yvon Thériault, parmi les hivernants en Floride.

Les procès-verbaux du CCFA étaient rédigés en français, en dépit de l'exigence de l'État floridien de tenir toute sa documentation en anglais ${ }^{129}$. Le CCFA prit rarement des mesures pour assurer le fait français dans son environnement, mais 1995 fut une année décisive pour le fait français en Amérique. Des préoccupations ne tardèrent pas à se manifester dès janvier: lors de sa rencontre du 17 janvier, le CA expliqua aux propriétaires du Casino Hall que «les directions [sic] à suivre [pour l'ascenseur] devraient être en français ${ }^{130}$ ». Ils cherchaient ainsi à améliorer l'accessibilité des lieux par la francisation. Le CA tint compte des commentaires émis au sujet de la musique à l'assemblée du 27 février. "À l'avenir, la musique devrait être planifi[ée], estimait-il, et la saison prochaine nous feron[s] l'essai d'alterner en [g]ardant Emil Sagna [un musicien anglophone] et un musicien de langue française Guy Harvey ${ }^{131} »$. On proposa d'ailleurs, durant la rencontre de novembre, de jouer l'hymne national ${ }^{132}$

125. Ibid.

126. Robert F. Harney, "The Palmetto and the Maple Leaf : Patterns of Canadian Migration to Florida», dans Randall M. Miller et George E. Pozzetta, Shades of the Sunbelt: Essays on Ethnicity, Race and the Urban South, Boca Raton, Florida Atlantic University Press, 21-39.

127. Gaétan Gervais, Des gens de résolution : le passage du "Canada français " à l' "Ontario français" (Sudbury, Prise de Parole, 2003); Yves Frenette, Brève histoire des Canadiens français (Montréal, Boréal, 1998), Marcel Martel, Le deuil d'un pays imaginé: rêves, luttes et déroute du Canada français: les rapports entre le Québec et la francophonie canadienne, 1867-1975 (Ottawa, Presses de l'Université d'Ottawa, 1997).

128. E.-Martin Meunier et Joseph Yvon Thériault, "Que reste-t-il de l’intention vitale du Canada français ?», dans Joseph Yvon Thériault, Linda Cardinal et Anne Gilbert, dir., L'espace francophone en milieu minoritaire au Canada (Montréal, Fides, 2008), 205.

129. M. Ouellette, Entrevue, op. cit., 5 janvier 2009.

130. "Assemblée 17 Jan 95 Chez Georgette Gamache», Club canadien-français d'Amérique/de Lake Worth 1994-2005, 12.

131. "Assemblée du 27 Février 95 Chez Albert Savoie», Club canadien-français d'Amérique/de Lake Worth 1994-2005, 16.

132. Le contexte du commentaire laisse entrevoir qu'il s'agit de l'ô Canada. 
à titre de chant rassembleur. La décision fut quand même prise quelques semaines seulement après le référendum sur la souveraineté du Québec. Les motivations n'étaient probablement pas anodines...

Au-delà des liens communautaires au CCFA, le Sud-Est floridien devenait une région de vie francophone fortuite. Jarvis observe que, durant les années 1980, "More French language services [were] offered in Southeast Florida than in many parts of officially bilingual Canada ${ }^{133}$.» Les antennes paraboliques permettaient de capter Radio-Canada, TVA et TQS. Les Canadiens français fréquentaient les bars, motels et magasins appartenant à des Québécois. Plusieurs ambulanciers, policiers, médecins, dentistes, garagistes, plombiers et nettoyeurs étaient francophones. Il était possible d'obtenir les quotidiens de Montréal et de Québec chaque jour sans compter la présence du Soleil de la Floride dès 1984. Un Club Richelieu vit le jour à Hollywood en 1979 tout comme le premier club de la Ligue nationale de hockey de la région, les Panthères de la Floride, dès $1994^{134}$. Tous les éléments incontournables des «Petits Canadas» (la mutuelle, la presse, l'église, les associations) étaient présents en Floride à l'exception de l'école, la grande lacune qui rendait le renouvellement de la communauté par la natalité pratiquement impossible.

Bien que la grande majorité de ces services était concentrée à Hollywood, il n'en restait pas moins possible de vivre exclusivement en français. Dans le Palm Beach Post, l'éditorialiste Robert Douglas écrivait en février 1988 qu'à Palm Beach «the extent of Canadian involvement is increasingly difficult to avoid, let alone ignore ${ }^{135}$ ” vu la présence des banques canadiennes (Desjardins, nationale, royale, etc.) et des entreprises comme Nortel, les millions de dollars investis dans l'immobilier tout comme la tenue à Palm Beach des matchs de pré-saison des Expos de Montréal. Bien qu'une liste des services disponibles en français à l'époque n'existe pas, nous en avons répertoriés 28 à Palm Beach pour le seul début $\mathrm{du} \mathrm{XXI}^{\mathrm{e}}$ siècle. Notons l'existence d'une messe catholique en français et la fondation d'une Communauté catholique d'expression française de Lake Worth en 1978. Cette messe affichait une volonté d'intégrer les Franco-Américains ne parlant plus le français et désireux de l'apprendre. À la messe où la lecture constitue une partie intégrale du rituel, Roger Groulx s'était, en effet, rendu compte, durant les années 1980, que certains

133. E. Jarvis, «Florida’s Forgotten Ethnic Culture...», loc. cit., 194.

134. R. Tremblay, Floribec..., op. cit., 41.

135. Robert Douglas, "The maple leaf and palm trees are intertwined», The Palm Beach Post (21 février 1988), Fonds Populations "Canadians in Florida », HSPBCA. 
Franco-Américains n'arrivaient pas à lire le Prions en Église. Comme il le faisait remarquer:

[Plusieurs Franco-Américains] avaient appris [le français] à la maison, mais l'école était anglaise, donc ils n'apprenaient jamais à le lire. D’où la raison qu'y en achetaient pas de Prions: y préféraient écouter. Y'en a d'entre eux qui peuvent lire des petites choses, mais pas pour aller lire en avant une lecture. $[\ldots][\mathrm{La}$ CCEFLW] organisait des rencontres de prières pendant une douzaine d'années. On faisait ça en français, puis on avait des familles de FrancoAméricains qui venaient] [...] écout[er] pour retrouver leurs racines. Y'aimaient ça entendre parler français. Y fallait pas parler trop vite, parce que là, y comprenaient] plus rien! [rires] $]^{136}$.

Si ce dénombrement démontre surtout l'existence d'une communauté ethnique très développée, on y comptait surtout des hivernants et des touristes qui maintenaient une «intention nationale» entre leur milieu de résidence canadien et leur demeure temporaire floridienne. En ce sens, la communauté, soutenue par les hivernants et les touristes, revêtait un caractère nationalitaire. Les immigrants profitaient sans doute de cet afflux de francophones qui leur permettaient de vivre un peu plus en français, même s'il n'existait pas d' "ambition nationale» chez eux. Cette dernière était plus l'apanage des résidants temporaires, plus désireux de reproduire une communauté spécifiquement francophone. L'élément nationalitaire souligne donc ces différences entre ceux qui font occasionnellement appel à la communauté et ceux qui maintenaient une vie en français et utilisaient ces associations ou établissements comme des prolongements de leur vie au Canada. Ouellette notait d'ailleurs son sentiment d'être chez lui «autant chez nous icitte [qu'au Canada,] pas de différence ${ }^{137}$ ». C'est en ses multiples rôles que la communauté revêtait un caractère nationalitaire: elle était tantôt exprimée comme une forme d'ethnicité et tantôt représentative d'une société à laquelle ses membres étaient issus. C'est en ce sens que la migration vers la Floride ne constitue plus forcément une contradiction de l'«ambition nationale» du Canada français. Il faudrait aussi ajouter que la communauté réussit à se renouveler grâce au flux constant de nouveaux migrants à l'inverse de plusieurs communautés ethniques aux États-Unis. 


\section{CONCLUSION}

Lorsque le CCFA se reconstitua en personne morale en 1996, il devint le Club canadien-français de Lake Worth ${ }^{138}$. Le nom s'ajustait à la nouvelle vocation de l'association. Alors qu'il desservait autrefois les Canadiens français habitant les États-Unis, le CCFA était devenu un pôle plus intimement attaché au Canada français et visait à desservir ses membres pendant leur absence du Canada. Ouellette quitta la présidence de l'organisme en $1997^{139}$. Le CCFA commença à diminuer ses services et la participation des membres en souffrit un peu. En 1997, la Floride canadiennefrançaise avait son heure de gloire derrière elle. La présence, la ferveur et la cohésion des Canadiens français n'auront jamais été aussi fortes. Des crimes contre des touristes canadiens, une hausse généralisée de la violence en Floride, le taux de change de moins en moins favorable aux Canadiens et la diversification des stations balnéaires antillaises ternirent l'image de la Floride et réduisirent son offre de services en français. Bien que les hivernants aient continué de la fréquenter en grand nombre, le renouvellement des effectifs était moins important et le taux de fréquentation touristique chuta de $32 \%$ entre 1992 et $1995^{140}$. Plusieurs établissements tels que le CCFA et le CCEFLW ne recrutèrent plus que le tiers de leurs anciens participants pendant la première décennie du $\mathrm{XXI}^{\mathrm{e}}$ siècle $^{141}$. La Floride demeura un point de convergence des francophones, mais elle perdit de son lustre et certains de ses services.

L'arrivée d'une masse d'hivernants et de vacanciers durant les années 1970 transforma l'état du fait français en Floride. Ces derniers migraient désormais entre deux îlots de l'Amérique française au lieu de la quitter. La croissance spectaculaire du nombre d'organismes et de services tout comme l'émergence d'une ambiance canadienne-française due à la mobilité constante entre le Canada central et la Floride de plusieurs hivernants

138. Note sans titre et sans date, ca. 1996; "Assemblée Mars 19/96 chez Georgette Gamache», "Assemblée chez Albert Savoie 27 Nov. 96 ", Club canadien-français d'Amérique/ de Lake Worth 19942005, 22, 31 ; Articles of Incorporation of Club canadien-francais de Lake Worth, Inc., 26 décembre 1996; Sandra B. Mortham, Secretary of State, Club canadien-francais de Lake Worth, Inc. Certificate, 26 décembre 1996, dans: ACCFLW.

139. "Assemblée Annuelle Dimanche, Février 16/97", dans Club canadien-français d'Amérique/ de Lake Worth 1994-2005, 35 ; "Assemblée Annuelle Dimanche, Février 16/97», 36; Élection 16 février 1997, dans ACCFLW.

140. S. de Repentigny, International Travel Survey..., op. cit.

141. Colette Beaudoin, Anne-Marie Groulx et Roger Groulx, Historique de la Communauté catholique d'expression française de Lake Worth, janvier 2008, 1, dans: Archives de la Communauté catholique d'expression française de Lake Worth. 
et touristes firent en sorte que ces communautés s'enchâssèrent, ne serait-ce que partiellement, au sein de l'espace francophone. Le changement de vocation du CCFA au tournant des années 1980 en témoigne bien. Au lieu de s'intégrer à la vie floridienne, l'organisme a cherché à maintenir la cohésion entre les Canadiens français à Palm Beach. Ces derniers retraités pouvaient désormais mettre à profit le revenu disponible, les régimes de retraite, l'amélioration de l'espérance de vie, les routes nouvelles et la chaleur floridienne sans obtenir la citoyenneté américaine tout en continuant de vivre en français.

La communauté a acquis un certain caractère "nationalitaire» pour devenir une francophonie nord-américaine en milieu minoritaire bien particulière. Elle se distinguait des autres puisqu'elle reposait presque entièrement sur la migration annuelle de Canadiens, que ces derniers n'élevaient pas d'enfants en Floride et qu'il n'y existait aucune école de langue française pour assurer la pérennité de la communauté sur place. Cette attraction de la Floride chez les Canadiens français depuis le XIX ${ }^{e}$ siècle représentait une intention qui était parfois en contradiction, parfois en concordance avec l'«intention nationale». Alors que plusieurs immigrants s'y étaient installés pour gagner leur vie ou même pour faire fortune dans le sud ensoleillé, cette région s'inscrivit dans la mémoire collective comme un lieu historique du Canada français. Il s'agirait, paradoxalement, d'une évasion dont on se souvient, ancrée dans la mémoire. «J'vais toujours hiverner ici, disait Ouellette, à moins qu'y me coupent les deux jambes au ras le corps!» Il ajoutait même : “J'ai bien plus peur de l'hiver que j'ai peur du diable ${ }^{142}$ !»

Prenant un peu plus de recul, la migration vers la Floride s'est distinguée des deux autres types de migrations canadiennes-françaises vers les États-Unis, soit l'émigration collective vers la Nouvelle-Angleterre pour y fonder de nouveaux îlots dans l'archipel de l'Amérique française ${ }^{143}$, soit l'émigration plus individuelle vers le Midwest ou la côte du Pacifique. La migration vers la Floride ressemblait d'abord aux migrations plus marginales vers les États de l'Ouest visant l'intégration à la société américaine, mais elle avait également des affinités avec la migration vacancière vers le Maine dont les plages attiraient bon nombre de touristes du Québec dès le milieu du XIX ${ }^{\mathrm{e}}$ siècle. Cependant, la Floride s'est différenciée avant

142. Maurice Ouellette, "Maurice Ouellette en Floride», Quelque part entre la 11 et la 17 (Sudbury, Première chaîne de Radio-Canada, 19 décembre 2006).

143. L'expression est celle de Dean Louder et d'Éric Waddell, Du continent perdu à l'archipel retrouvé: le Québec et l'Amérique française (Québec, Les Presses de l’Université Laval, 1983). 
tout en raison de l'afflux massif de touristes après la Deuxième Guerre mondiale et du contingent impressionnant d'hivernants après les années 1970 qui l'ont fréquentée année après année. La Floride représente donc le premier exemple d'une migration de masse canadienne-française en période contemporaine. 